\title{
Fatores associados à gravidade das reações transfusionais ocorridas em hospital de ensino, na cidade de São Paulo, entre 2007-2019
}

\section{Factors associated with the severity of transfusion reactions that occurred in a teaching hospital, in the city of São Paulo, between 2007-2019}

João Luiz Grandi,IV,* iD

Kelsy Catherina Nema Areco" iD

Akemi Chiba"I (iD)

Maria Melca Barros de Oliveirall' iD

Dulce Aparecida Barbosalv
I Hospital São Paulo, São Paulo, SP, Brasil

" Departamento de Informática em Saúde, Universidade Federal de São Paulo (Unifesp), São Paulo, SP, Brasil

III Hemocentro, Hospital São Paulo, Universidade Federal de São Paulo (Unifesp), São Paulo, SP, Brasil

Iv Escola Paulista de Enfermagem, Universidade Federal de São Paulo (Unifesp), São Paulo, SP, Brasil

* E-mail: jgrandi@huhsp.org.br

\section{RESUMO}

Introdução: Reações transfusionais podem acarretar sérias consequências aos receptores de hemocomponentes. Objetivo: Analisar a proporção de reações adversas quanto ao grau de severidade observado nas transfusões de sangue e identificar os fatores associados à gravidade dos incidentes transfusionais imediatos. Método: Estudo longitudinal retrospectivo de avaliação epidemiológica das reações transfusionais imediatas ocorridas entre 2007-2019 em hospital de ensino. Resultados: Das 332.222 transfusões sanguíneas administradas, foram reportadas 1.448 notificações de reações transfusionais imediatas. A média de incidência de reação transfusional foi de 4,4 por mil/ano. As reações moderadas e graves representam $13,5 \%$ do total dos eventos. Hemoglobulina e hematócrito não apresentaram distribuição normal ( $\mathrm{p} \leq 0,001)$. Conclusões: A análise dos dados possibilitou avaliar a prática transfusional na instituição, que se mostrou adequada, refletindo os constantes esforços para retroalimentar o Sistema Nacional de Hemovigilância. A gravidade das reações está associada à unidade de internação, sendo mais frequentes as graves nas unidades críticas e semicríticas, porém o estudo demonstrou que não se deve negligenciar as reações leves.

PALAVRAS-CHAVE: Gestão de Riscos; Reação Transfusional; Segurança do Paciente; Segurança do Sangue; Vigilância Sanitária

\section{ABSTRACT}

Introduction: Transfusion reactions can have serious consequences for recipients of blood components. Objective: To analyze the proportion of adverse reactions regarding the degree of severity observed in transfusions and to identify factors associated with the severity of immediate transfusion incidents. Method: This is a retrospective longitudinal study of epidemiological evaluation regarding the immediate transfusion reactions that occurred between 2007 and 2019 in a teaching hospital. Results: Of the 332,222 blood transfusions administered in the period, 1,448 notifications of immediate transfusion reactions were reported. The average annual incidence of transfusion reaction was 4.4 per thousand. Moderate and severe reactions represent $13.5 \%$ of all events. Hemoglobin and hematocrit did not present regular distribution $(p \leq 0.001)$. Conclusion: The data analysis made it possible to evaluate the transfusion practice at the institution, which proved to be adequate and reflected the constant efforts to feed the Brazilian System of Hemovigilance. The severity of the reactions has been associated with inpatient units, which are more severe in the critical and semi-critical care units. However, the study showed that mild reactions should not be neglected.

KEYWORDS: Risk Management; Transfusion Reaction; Patient Safety; Blood Safety; Health Surveillance 


\section{INTRODUÇÃO}

As transfusões de sangue e de hemocomponentes são intervenções terapêuticas que salvam vidas e melhoram a saúde dos pacientes, especialmente daqueles que estão hospitalizados. A maioria das transfusões fornece os benefícios esperados de substituição segura e efetiva de componentes sanguíneos ${ }^{1}$. Contudo, esse procedimento não é livre de riscos. Durante a transfusão, podem ocorrer transmissão de agentes infecciosos e complicações clínicas ocasionadas por eventos adversos ${ }^{1,2}$. 0 risco de reações transfusionais é estimado em 1:16.500, sendo que a ocorrência de óbitos por indicação errada de transfusão de sangue gira em torno de 1:500.000³.

O componente de sangue mais administrado na prática clínica é o concentrado de hemácias, indicado para o tratamento das anemias ${ }^{1,2,3,4}$. Dos pacientes internados em unidades críticas, estima-se que cerca de $70 \%$ apresentem anemia e, destes, $25 \%$ vão receber hemotransfusão $0^{5,6}$.

Os valores de hemoglobina $(\mathrm{Hb}), \mathrm{em} \mathrm{g/dL}$, e de hematócrito $(\mathrm{Ht})$ auxiliam a conduta em hemoterapia. Valores baixos tanto de $\mathrm{Hb}$ quanto de $\mathrm{Ht}$ podem indicar anemia. É preconizada a transfusão de concentrado de hemácias em pacientes críticos com $\mathrm{Hb}$ abaixo de $7 \mathrm{~g} / \mathrm{dL}$. A transfusão de sangue não está indicada quando a $\mathrm{Hb}$ é $>9,0 \mathrm{~g} / \mathrm{dL}^{7,8}$.

Vários profissionais da saúde atuam para garantir a segurança desse procedimento. As agências transfusionais/bancos de sangue verificam a compatibilidade sanguínea e realizam testes imuno-hematológicos, pré e pós-transfusional, para minimizar riscos e reações adversas. Cada serviço de saúde pode seguir protocolos diferentes para a administração de hemoterapia, porém é recomendado que o paciente seja monitorado durante os primeiros $15 \mathrm{~min}$ da infusão para observância de sinais e sintomas indicativos de eventos adversos, tais como tremores, cianose, reações urticariformes, dor torácica ou lombar, hipotensão, choque, urina escura, taquicardia e febre. Os efeitos adversos de uma transfusão nem sempre são evitáveis e ocorrem em $3 \%$ a $10 \%$ dos procedimentos ${ }^{2}$.

As reações transfusionais podem ser classificadas de acordo com a gravidade, em leve, moderada, grave e evento sentinela, e com o tempo de seu aparecimento, em imediato ou tardio. As complicações agudas, que ameaçam a vida, são mais raras, enquanto as reações leves são mais comuns. Estudos mostram que as reações transfusionais notificadas à Agência Nacional de Vigilância Sanitária (Anvisa), em sua maioria, são reações leves imediatas ${ }^{1,4,5}$.

Reações transfusionais imediatas são aquelas que ocorrem durante ou em até $24 \mathrm{~h}$ depois de instalada a infusão sanguínea. Sua gravidade pode variar bastante nesse período, embora a maioria das reações com severidade elevada e ameaça à vida geralmente ocorra no início da administração dos componentes sanguíneos ${ }^{10}$. Estima-se que a ocorrência de óbitos como resultado da utilização de um componente de sangue transfundido incorretamente é de 1:500.000 como consequência de reações imediatas 9

Os serviços de hemovigilância que avaliam a terapia transfusional visam garantir a segurança do paciente, diminuindo os erros de prescrição de hemocomponentes, melhorando a detecção de eventos adversos e prevenindo sua recorrência, além de contribuir para a melhoria da qualidade do ciclo do sangue, considerando todo o processo que envolve o doador e o receptor. Contudo, as estatísticas relativas à hemovigilância ainda não estão amplamente disponíveis no Brasil ${ }^{11}$.

Os serviços de hemovigilância contribuem com propostas de implantação de medidas preventivas e corretivas baseadas no conhecimento das características dos pacientes e dos fatores associados aos eventos adversos. 0 gerenciamento de risco ${ }^{5} \mathrm{em}$ hemovigilância consiste em estabelecer estratégias de acompanhamento e monitoramento da hemotransfusão para evitar a recorrência de eventos adversos e minimizar a gravidade quando estes ocorrem. Portanto, dados relativos à hemovigilância subsidiam a tomada de decisão voltada ao gerenciamento de riscos.

Nesse contexto e com base nos dados disponíveis coletados rotineiramente no serviço de hemovigilância de um hospital universitário de alta complexidade, o presente estudo teve como objetivo analisar os incidentes transfusionais imediatos, quanto ao grau de severidade e à proporção de reações adversas observadas nas transfusões, e identificar os fatores associados à gravidade desses incidentes.

\section{MÉTODO}

Desenho, período e local do estudo

Trata-se de um estudo longitudinal retrospectivo de avaliação epidemiológica das reações transfusionais imediatas ocorridas entre 2007 e 2019 em um hospital de ensino de alta complexidade. De caráter filantrópico, o hospital conta com 862 leitos, é integrante da Rede Brasileira de Hospitais Sentinela desde 2002, localizado na cidade de São Paulo (SP) e é referência nacional e internacional para o ensino e a pesquisa. Os dados foram coletados por meio das fichas de notificação de reações transfusionais, recebidas e armazenadas em banco de dados do Microsoft Excel, pela Gerência de Risco, no período de maio de 2007 a dezembro de 2019.

População e amostra

Os dados do estudo foram obtidos de um total de 1.448 fichas de notificações de incidentes transfusionais (FNIT), usadas para alimentação do Sistema Nacional de Hemovigilância (SNH), e mantidas pelo Hemocentro e pela Gerência de Risco, que realizam as ações de hemovigilância no Hospital São Paulo, Hospital Universitário da Universidade Federal de São Paulo (Unifesp).

\section{Critério de inclusão}

Todas as FNIT de pacientes que receberam transfusão de sangue e/ou de seus derivados, reportando incidente transfusional imediato por qualquer causa, que continham, na requisição de transfusão, os valores devidamente registrados de $\mathrm{Hb}$ e $\mathrm{Ht}$. 


\section{Critério de exclusão}

Foram excluídas as FNIT com sinais e sintomas não compatíveis com reações transfusionais imediatas e aquelas com diagnóstico de reação tardia. As FNIT com diagnóstico de reação transfusional provável ou possível também não foram incluídas neste estudo. Foram excluídas as FNIT anteriores a 2007, nas quais ainda não era realizada a pesquisa dos valores de $\mathrm{Hb}$ e $\mathrm{Ht}$ para anotação na FNIT pela Gerência de Risco.

\section{Protocolo do estudo}

A ocorrência dos incidentes transfusionais foi registrada nas FNIT, elaboradas e validadas pela própria instituição para alimentação do SNH da Anvisa. As FNIT fazem parte da rotina de hemovigilância da instituição pesquisada. Para a análise deste estudo, foram utilizadas as seguintes variáveis das FNIT: data de nascimento, sexo, data da transfusão, tipagem do receptor (sistema ABO e fator Rh), unidade de internação, valores de $\mathrm{Hb}$ e $\mathrm{Ht}$, tipo de hemocomponente administrado e manifestações clínicas referidas, gravidade e classificação da reação. Depois de confirmados o diagnóstico e a classificação das reações por médico hematologista no Hemocentro da instituição, as FNIT seguem para a Gerência de Risco para inclusão no banco de dados da instituição e posterior inserção no SNH.

As notificações dos incidentes transfusionais (ou reações adversas) seguiram o padrão estabelecido na instituição: depois de identificados sinais e/ou sintomas compatíveis com incidentes transfusionais, a infusão é interrompida e novas amostras de sangue são coletadas para envio à Agência Transfusional para testes imuno-hematológicos confirmatórios do diagnóstico da reação. A classificação dos incidentes transfusionais quanto ao diagnóstico foi confirmada por médico hemoterapeuta, seguindo as recomendações da Anvisa publicadas em $2015^{2}$.

Os testes pré-transfusionais, $\mathrm{Hb}$ e $\mathrm{Ht}$ foram realizados no Laboratório de Análises Clínicas do Hospital São Paulo e seguiram as recomendações definidas pelo Mayo Clinical Medical Laboratories ${ }^{\circledR}$, considerando que os critérios de normalidade para os valores de $\mathrm{Hb}$ e Ht são ajustados de acordo com o sexo e a idade. Assim, para maiores de 16 anos, os valores de $\mathrm{Hb}$ considerados normais foram 13,5-17,5 g/dL para homens e 12,0-15,5 g/dL para mulheres; os valores de $\mathrm{Ht}$ foram $39 \%-50 \%$ e $35 \%-45 \%$ para homens e mulheres, respectivamente. Já para crianças e adolescentes até 16 anos, os valores normais de $\mathrm{Hb}$ variam de 11,0-14,5 a 13,5-17,5 g/dL entre os meninos e de 12,0-15,0 a 12,0-15,5 g/dL entre as meninas; os valores de $\mathrm{Ht}$ variam de $33 \%-43 \%$ a $39 \%-50 \%$ para os meninos e de $35 \%-44 \%$ a $35 \%-45 \%$ para as meninas.

\section{Análise dos resultados}

Para a análise dos dados, as variáveis categóricas foram descritas por frequência absoluta e relativa. As variáveis numéricas foram descritas por medidas de tendência central (média e mediana) e variabilidade (mínimo, máximo, desvio-padrão, intervalo interquartil) e examinadas quanto à aderência à distribuição normal por meio do teste de Kolmogorov-Smirnov. A associação entre variáveis categóricas independentes foi estudada pelo teste $x^{2}$ de Pearson e entre variáveis numéricas e categóricas pelo teste de Mann-Whitney. Em todos os testes, o nível de significância estabelecido foi de $5 \%$. Para realização de todas as análises, os dados originais registrados em planilha Microsoft Excel $₫ 2010$ foram importados para o pacote estatístico SPSS $®$ v. 22.

\section{Aspectos éticos}

Este estudo, depois de inserido na Plataforma Brasil, foi avaliado e aprovado sob parecer $n^{\circ} 1794086$ e Certificado de Apresentação para Apreciação Ética (CAAE) no 160855416.5.0000.5505, emitido pelo Comitê de Ética em Pesquisa da Unifesp, em 26 de outubro de 2016. A presente investigação atende aos preceitos éticos da Resolução n 466, de 12 de dezembro de 2012, do Conselho Nacional de Saúde.

\section{RESULTADOS}

De um total de 1.663 FNIT por todas as causas, foram excluídas 215 por não apresentarem os valores de $\mathrm{Hb}$ e $\mathrm{Ht}$, restando 1.448 fichas para análise.

Nos 13 anos em estudo, de 2007 a 2019, foram realizadas 332.222 transfusões de sangue e derivados na instituição investigada, das quais 1.448 apresentaram reação transfusional imediata, representando 4,4 por 1.000 transfusões em média ao ano. Nesse período, o número de transfusões por ano oscilou de 39.300 em 2014 para 18.016 em 2016, com média de 25.560 transfusões/ano (Tabela 1).

Nas FNIT verificadas, a proporção de homens $(51,2 \%)$ e de mulheres $(48,9 \%)$ foi semelhante, correspondendo a 740 e 708 indivíduos, respectivamente (Tabela 2 ).

Em relação às unidades notificadoras, o maior percentual de ocorrências foi registrado nas unidades de pacientes oncológicos $(35,7 \%)$ e o menor nas unidades cirúrgicas e obstétricas $(8,0 \%)$ (Tabela 2$)$.

Tabela 1. Frequência absoluta e relativa de reações transfusionais imediatas e número de transfusões realizadas por ano $(n=1.448)$. Hospital São Paulo, 2007-2019.

\begin{tabular}{lccc}
\hline \multirow{2}{*}{ Ano } & \multicolumn{2}{c}{$\begin{array}{c}\text { Reações transfusionais } \\
\text { imediatas }\end{array}$} & $\begin{array}{c}\text { Transfusões de } \\
\text { hemocomponentes }\end{array}$ \\
\cline { 2 - 4 } & $\mathbf{N}^{\circ}$ & $\%$ & Total $\left.\mathbf{N}^{\circ}\right)$ \\
\hline 2007 & 127 & 5,3 & 23.926 \\
2008 & 63 & 2,2 & 29.234 \\
2009 & 88 & 3,5 & 25.130 \\
2010 & 133 & 4,8 & 27.873 \\
2011 & 104 & 3,3 & 31.179 \\
2012 & 114 & 3,9 & 29.347 \\
2013 & 132 & 4,0 & 33.173 \\
2014 & 184 & 4,7 & 39.300 \\
2015 & 124 & 4,7 & 26.504 \\
2016 & 139 & 7,5 & 18.618 \\
2017 & 68 & 2,7 & 24.304 \\
2018 & 105 & 4,4 & 23.789 \\
2019 & 135 & 5,5 & 24.249 \\
\hline Total & 1.448 & 4,2 & 332.222 \\
\hline
\end{tabular}

Fonte: Elaborada pelos autores, 2019. 
Quanto ao tipo de hemocomponente, o concentrado de hemácias correspondeu a $72,3 \%$, as plaquetas a $21,3 \%$ e os demais hemocomponentes a 6,4\% do total das FNIT (Tabela 2). Na instituição estudada, o total de transfusões por concentrado de hemácias no período foi próximo de 45,0\% (dado não apresentado).

Já em relação ao sistema $\mathrm{ABO}$ e ao fator $\mathrm{Rh}$, os tipos sanguíneos $\mathrm{O}^{(+)}$e $\mathrm{A}^{(+)}$são os mais frequentes nas FNIT, com $43,5 \%$ e $31,8 \%$, respectivamente. Entre os menos representativos, encontram-se os grupos $A^{(-)}$, com $1,2 \%$, e os grupos $B^{(-)}$e $A B^{(-)}$, com cerca de $0,9 \%$ cada do total da amostra (Tabela 2).

As variáveis numéricas idade e valor de $\mathrm{Hb}$ e $\mathrm{Ht}$ não apresentaram distribuição normal $(p<0,001)$. A idade mediana observada foi de 45 anos (intervalo interquartil $=35$ ), variando de 0 (menor que 1 ano) a 94 anos completos. $\mathrm{O}$ valor mediano de $\mathrm{Hb}$ foi $7,9 \mathrm{mg} / \mathrm{dL}$ (intervalo interquartil = 2,1), com mínimo de 2,4 e máximo de 17,6; os valores de $\mathrm{Ht}$ variaram de $7,7 \%$ a $52,3 \%$, com mediana de 23,5\% (intervalo interquartil $=5,8$ ).

Na Tabela 3 são apresentados os dados quanto à gravidade das reações transfusionais. Verifica-se que $86,5 \%$ das FNIT foram classificadas como grau I de severidade. As de grau II e III corresponderam a $13,5 \%$ do total. De acordo com a classificação da reação transfusional, o tipo mais comum diagnosticado foi a reação febril não hemolítica (RFNH), que representa 57,2\% das FNIT, seguida da reação

Tabela 2. Caracterização das fichas de notificações de incidentes transfusionais (FNIT) $(n=1.448)$. Hospital São Paulo, 2007-2019.

\begin{tabular}{|c|c|c|c|}
\hline Variáveis & & $\mathbf{N}^{\circ}$ & $\%$ \\
\hline \multirow[t]{2}{*}{ Sexo } & Masculino & 740 & 51,2 \\
\hline & Feminino & 708 & 48,8 \\
\hline \multirow[t]{6}{*}{ Unidade de Internação } & Oncológicas & 517 & 35,7 \\
\hline & Clínicas médicas & 249 & 17,2 \\
\hline & Urgência e emergência & 229 & 15,8 \\
\hline & Críticas e semicríticas & 220 & 15,2 \\
\hline & Pediátricas & 118 & 8,1 \\
\hline & Cirúrgicas e obstétricas & 115 & 8,0 \\
\hline \multirow[t]{4}{*}{ Hemocomponentes } & Concentrado de hemácias & 1.047 & 72,3 \\
\hline & Plaquetas & 308 & 21,3 \\
\hline & Plasma fresco & 91 & 6,2 \\
\hline & Hemoglobina/granulócitos & 2 & 0,2 \\
\hline \multirow[t]{8}{*}{$\mathrm{ABO} /$ Fator Rh } & A- & 17 & 1,2 \\
\hline & $A+$ & 461 & 31,8 \\
\hline & $B-$ & 14 & 0,9 \\
\hline & $\mathrm{B}+$ & 160 & 11,1 \\
\hline & $A B-$ & 13 & 0,9 \\
\hline & $\mathrm{AB}+$ & 60 & 4,1 \\
\hline & $0-$ & 94 & 6,5 \\
\hline & $0+$ & 629 & 43,5 \\
\hline \multirow[t]{2}{*}{ Hemoglobina } & Abaixo do normal & 1.411 & 97,5 \\
\hline & Normal & 37 & 2,5 \\
\hline \multirow[t]{3}{*}{ Hematócrito } & Abaixo do normal & 1.413 & 97,6 \\
\hline & Acima do normal & 4 & 0,2 \\
\hline & Normal & 31 & 2,2 \\
\hline
\end{tabular}

Fonte: Elaborada pelos autores, 2019. alérgica (ALG), com 37,5\% do total de casos. Em proporções menores, foram observadas a sobrecarga circulatória (TACO), com 2,6\%; a reação hemolítica aguda imunológica (RHAl), com 1,2\%, e a lesão pulmonar aguda (TRALI), com cerca de $1,0 \%$ das reações. Com valores ainda menos significativos foram observadas as reações: dispneia associada à transfusão (DAT), contaminação bacteriana (CB) e hipotensão relacionada à transfusão (HIPOT), totalizando $0,5 \%$ dos casos reportados. Os principais sinais e sintomas observados foram as lesões de pele, com $27,3 \%$, o prurido, com $26,9 \%$, e a taquicardia, com $16,9 \%$ de casos relatados nas FNIT. A hipertermia (elevação da temperatura corporal em pelo menos $1^{\circ} \mathrm{C}$ ) foi referida em apenas 63 casos, representando 4,3\% do total das FNIT (Tabela 3).

Somente a unidade notificadora apresentou associação estatisticamente significante com o grau de gravidade, destacando-se as unidades críticas e semicríticas em relação às demais, com proporção de reações de gravidade moderada/grave igual a $13,5 \%$ $(p=0,001)$ (Tabela 4). As variáveis sexo $(p=0,847)$, sistema ABO $(p=0,646)$ e fator $\operatorname{Rh}(p=0,852)$ não apresentaram associação com o grau de gravidade pelos testes $x^{2}$ de Pearson.

Tabela 3. Classificação das informações presentes nas fichas de notificações de incidentes transfusionais (FNIT) de acordo com a gravidade e a tipologia e sinais e sintomas das reações transfusionais $(\mathrm{n}=1.448)$. Hospital São Paulo, 2007-2019.

\begin{tabular}{|c|c|c|c|}
\hline & & $\mathrm{N}^{\circ}$ & $\%$ \\
\hline \multirow[t]{3}{*}{ Gravidade } & Leve (grau I) & 1.253 & 86,5 \\
\hline & Moderada (grau II) & 149 & 10,3 \\
\hline & Grave (grau III) & 46 & 3,2 \\
\hline \multirow[t]{9}{*}{ Tipo de reação* } & Febril não hemolítica (RFNH) & 828 & 57,2 \\
\hline & Alérgica (ALG) & 543 & 37,5 \\
\hline & Sobrecarga circulatória (TACO) & 38 & 2,6 \\
\hline & $\begin{array}{l}\text { Hemolítica aguda } \\
\text { imunológica (RHAl) }\end{array}$ & 15 & 1,2 \\
\hline & Lesão pulmonar aguda (TRALI) & 12 & 1,0 \\
\hline & $\begin{array}{l}\text { Dispneia associada à } \\
\text { transfusão (DAT) }\end{array}$ & 3 & 0,2 \\
\hline & Contaminação bacteriana (CB) & 2 & 0,2 \\
\hline & $\begin{array}{l}\text { Hipotensão relacionada à } \\
\text { transfusão (HIPOT) }\end{array}$ & 1 & 0,1 \\
\hline & Calafrios & 399 & 33,0 \\
\hline \multirow[t]{14}{*}{ Sinais e Sintomas } & Calafrios & 399 & 27,5 \\
\hline & Lesões de pele & 396 & 27,3 \\
\hline & Prurido & 390 & 26,9 \\
\hline & Taquicardia & 245 & 16,9 \\
\hline & Dispneia & 183 & 12,6 \\
\hline & Elevação da temperatura $=>1^{\circ} \mathrm{C}$ & 63 & 4,3 \\
\hline & Alteração da pressão arterial & 55 & 3,8 \\
\hline & Dor torácica & 35 & 2,7 \\
\hline & Cianose & 31 & 2,5 \\
\hline & Dor lombar & 25 & 2,1 \\
\hline & Icterícia & 9 & 0,6 \\
\hline & Hematúria & 4 & 0,2 \\
\hline & Hipertensão & 4 & 0,3 \\
\hline & Outros & 73 & 5,0 \\
\hline
\end{tabular}

Fonte: Elaborada pelos autores, 2019.

*Em cada FNIT podem ser reportados mais de um sinal ou sintoma. 
Tabela 4. Distribuição das informações presentes nas fichas de notificações de incidentes transfusionais (FNIT) como sexo, sistema ABO, fator Rh e unidade de ocorrência de acordo com a gravidade das reações transfusionais ( $n=1.448)$. Hospital São Paulo, $2007-2019$.

\begin{tabular}{|c|c|c|c|c|c|c|c|c|}
\hline & & \multicolumn{4}{|c|}{ Gravidade (grau)* } & \multirow{2}{*}{\multicolumn{2}{|c|}{ Total }} & \multirow{3}{*}{ Teste $x^{2}$} \\
\hline & & \multicolumn{2}{|c|}{1} & \multicolumn{2}{|c|}{ II e III } & & & \\
\hline & & $\mathrm{N}^{\circ}$ & $\%$ & $\mathrm{~N}^{\circ}$ & $\%$ & $\mathrm{~N}^{\circ}$ & $\%$ & \\
\hline \multirow[t]{2}{*}{ Sexo } & Feminino & 639 & 86,6 & 101 & 13,6 & 740 & 100,0 & \multirow{2}{*}{$p=0,847$} \\
\hline & Masculino & 615 & 86,7 & 94 & 13,3 & 708 & 100,0 & \\
\hline \multirow[t]{4}{*}{$\mathrm{ABO}$} & $A$ & 419 & 87,7 & 59 & 12,3 & 478 & 100,0 & \multirow{4}{*}{$p=0,646$} \\
\hline & B & 146 & 83,9 & 28 & 16,1 & 174 & 100,0 & \\
\hline & $A B$ & 60 & 82,2 & 13 & 17,8 & 73 & 100,0 & \\
\hline & 0 & 626 & 86,6 & 97 & 13,4 & 723 & 100,0 & \\
\hline \multirow[t]{3}{*}{ Fator Rh } & Negativo & 120 & 87,0 & 18 & 13,0 & 138 & 100,0 & \multirow{3}{*}{$p=0,852$} \\
\hline & Positivo & 1.131 & 86,3 & 179 & 13,7 & 1.310 & 100,0 & \\
\hline & Cirúrgicas e obstétricas & 101 & 87,8 & 14 & 12,2 & 115 & 100,0 & \\
\hline \multirow[t]{5}{*}{ Unidade de internação } & Clínicas médicas & 217 & 87,2 & 32 & 12,8 & 249 & 100,0 & \multirow{5}{*}{$p=0,001$} \\
\hline & Críticas e semicríticas & 167 & 76,0 & 53 & 24,0 & 220 & 100,0 & \\
\hline & Oncológicas & 463 & 89,7 & 54 & 10,3 & 517 & 100,0 & \\
\hline & Pediátricas & 101 & 85,6 & 17 & 14,4 & 118 & 100,0 & \\
\hline & Urgência e emergência & 201 & 88,1 & 28 & 11,9 & 229 & 100,0 & \\
\hline Total & & 1.250 & 85,7 & 198 & 14,3 & 1.448 & 100,0 & \\
\hline
\end{tabular}

Fonte: Elaborada pelos autores, 2019.

${ }^{*}$ Grau I = leve; II = moderada; III = grave.

Comparando-se a idade e os valores de $\mathrm{Hb}$ e $\mathrm{Ht}$ nos dois grupos de grau de severidade leve e moderada/grave das FNIT, os valores medianos de $\mathrm{Hb}$ e $\mathrm{Ht}$ foram semelhantes nos dois grupos, enquanto a idade mediana do grupo que apresentou reação leve (43 anos) foi menor que no grupo com reação moderada ou grave (51 anos), com $p=0,003$ (Tabela 5).

\section{DISCUSSÃO}

Neste estudo, analisaram-se as FNIT que ocorreram e foram reportados rotineiramente à Anvisa, nos últimos 13 anos, em hospital de ensino de alta complexidade. De 2007 a 2019, foram examinadas 1.448 FNIT de um total de 1.663 reações imediatas, referentes a 332.222 transfusões de sangue e de derivados, realizadas na instituição investigada. No estado de São Paulo, em 2016, os incidentes imediatos representaram $99 \%$ do total das reações transfusionais reportadas ao Centro de Vigilância Sanitária (CVS) ${ }^{12}$.
A taxa de incidência anual encontrada no presente estudo, de 4,4 reações por 1.000 transfusões, foi menor que a encontrada no Hospital de Clínicas do Uruguai, onde a taxa foi de 8,3 por 1.000 hemocomponentes administrados ${ }^{13}$. Taxas inferiores foram encontradas com valores de 1,7 no norte de Minas Gerais ${ }^{14}$ e de 3,9 por 1.000 hemotransfusões no norte do Paraná ${ }^{15}$. Outros estudos fora do Brasil relataram taxas anuais de incidência de reações adversas entre 0,2 e 7,7 por 1.000 hemocomponentes infundidos ${ }^{10,16,17}$. Tais valores podem variar de acordo com as estratégias adotadas em cada serviço para hemovigilância, como a busca ativa ou passiva ${ }^{17}$.

Nas FNIT analisadas, a proporção de homens $(51,2 \%)$ e de mulheres $(48,9 \%)$ receptores de sangue e hemocomponentes foi semelhante, dados corroborados por outros estudos ${ }^{6,18,19}$. A idade mediana de 45 anos (0-94 anos) foi semelhante à idade média de pacientes que apresentaram reações transfusionais em hospital no Nordeste

Tabela 5. Distribuição das informações presentes nas fichas de notificações de incidentes transfusionais (FNIT) como idade (anos), valores da Hemoglobina (mg/dl) e do hematócrito (\%) de acordo com a gravidade das reações transfusionais ( $\mathrm{n}=1.448)$. Hospital São Paulo, $2007-2019$.

\begin{tabular}{|c|c|c|c|c|c|c|}
\hline \multirow{2}{*}{ Gravidade** } & \multicolumn{2}{|c|}{ Idade (anos)* } & \multicolumn{2}{|c|}{ Hemoglobina $(\mathrm{mg} / \mathrm{dl})^{*}$} & \multicolumn{2}{|c|}{ Hematócrito (\%)* } \\
\hline & 1 & II e III & 1 & II e III & 1 & II e III \\
\hline$N$ & 1.045 & 163 & 1.045 & 163 & 1.045 & 163 \\
\hline Média & 42,9 & 48,6 & 8,1 & 8,2 & 24,0 & 24,6 \\
\hline Mediana & 43,0 & 51,0 & 7,9 & 7,9 & 23,4 & 23,7 \\
\hline Desvio-padrão & 20,95 & 24,57 & 1,74 & 1,99 & 5,19 & 5,97 \\
\hline Mínimo & 0,0 & 0,0 & 2,4 & 3,4 & 7,7 & 9,7 \\
\hline Máximo & 94,0 & 93,0 & 17,6 & 15,0 & 52,3 & 43,7 \\
\hline Intervalo interquartil & 34,0 & 40,0 & 2,1 & 2,0 & 5,7 & 6,0 \\
\hline Teste de Mann-Withney & \multicolumn{2}{|c|}{$p=0,003$} & \multicolumn{2}{|c|}{$p=0,470$} & \multicolumn{2}{|c|}{$p=0,453$} \\
\hline
\end{tabular}

Fonte: Elaborada pelos autores, 2019.

* Variáveis não apresentaram distribuição normal pelo teste de Kolmogorov-Smirnov.

** Grau I = leve; II = moderada; III = grave. 
brasileiro (média de 48 anos), que também incluiu pacientes pediátricos. Outra publicação que não incluiu pacientes pediátricos apresentou médias de idade maiores, de 55 a 61 anos $^{5,20,21}$. A mediana de idade encontrada na presente análise está dentro da faixa etária em que ocorreu a maior frequência de reações transfusionais no estado de São Paulo ( 30 a 70 ou mais anos) $)^{12}$.

Em relação ao tipo sanguíneo dos receptores identificados nas FNIT, os tipos $\mathrm{O}^{(+)}$e $\mathrm{A}^{(+)}$são os mais frequentes, com proporções em torno de $43,0 \%$ e $32,0 \%$, respectivamente. Essas proporções são semelhantes às de doadores de sangue no Brasil, que, em 2015, corresponderam a $43,0 \%$ para o grupo $\mathrm{O}^{(+)}$e $30,3 \%$ para o $\mathrm{A}^{(+) 22}$.

Com base nos valores de $\mathrm{Hb}$ e $\mathrm{Ht}$, pode-se dizer que a prática transfusional na instituição pesquisada está de acordo com as diretrizes preconizadas, uma vez que $97,5 \%$ das transfusões ocorreram em pacientes com valores abaixo dos parâmetros de referência normal. Em estudo brasileiro que analisou transfusões em pacientes críticos de diferentes unidades de terapia intensiva, no qual metade das indicações para transfusão aconteceram por $\mathrm{Hb}$ baixa e $32,0 \%$ por sangramentos ativos, a prática transfusional foi considerada parcialmente adequada ${ }^{8}$. 0 limite inferior dos valores de referência normais para a concentração de $\mathrm{Hb}$ no sangue tem sido objeto de pesquisa por autores norte-americanos preocupados com o diagnóstico e a prevalência da anemia ${ }^{23}$.

As unidades de internação oncológicas representaram 37,5\% do total das FNIT analisadas. Quanto à utilização do hemocomponente, $72,3 \%$ das reações ocorreram com a administração do concentrado de hemácias, que é o hemoderivado comumente empregado na prática clínica ${ }^{1,5,6,24}$; dados ligeiramente menores do que o observado no estado de São Paulo entre os anos de 2012 e 2015, nos quais foram realizadas $3.384 .818(56,0 \%)$ de concentrado de hemácias do total de 6.045 .102 transfusões. 0 fato de que $72,2 \%$ das reações imediatas na instituição pesquisada ocorreram por transfusão do concentrado de hemácias é indicativo da associação entre esse componente e os incidentes transfusionais imediatos. Não foram analisadas reações fatais no período deste estudo.

De acordo com os resultados apresentados, a proporção de reações adversas imediatas de maior gravidade (grau II e III) foi de $13,5 \%$. Tais reações representam potencial risco de morte para o paciente ${ }^{18}$. Em estudo realizado na cidade de Havana, em Cuba, com 3.347 hemotransfusões e 40 reações transfusionais, os autores encontraram $8,0 \%$ de reações graves com dois óbitos, ambos reportados em unidade de terapia intensiva ${ }^{25}$.

Nas FNIT analisadas, as reações mais comuns foram a reação febril não hemolítica $(57,2 \%)$ e a reação alérgica $(37,5 \%)$, semelhantes aos dados reportados em outros dois estudos nacionais ${ }^{10,24}$. Em investigação conduzida no Uruguai ${ }^{13}$ com 58 reações transfusionais, os autores evidenciaram sete (12\%) reações de severidade grave (grau I) e duas que resultaram em óbito dos pacientes.

Os principais sinais e sintomas reportados nas FNIT foram; calafrios $(27,5 \%)$, lesões de pele $(27,3 \%)$, prurido $(26,9 \%)$ e taquicardia $(16,9 \%)$. A hipertermia com elevação de pelo menos $1^{\circ} \mathrm{C}$ da temperatura corpórea foi reportada em 4,3\% das reações, achados estes compatíveis com a literatura ${ }^{1,5,6}$. Já as principais manifestações clínicas foram urticária $(40,4 \%)$ e calafrios $(20,2 \%)^{1}$. No Japão ${ }^{19}$, entre as reações mais graves, os autores reportaram sinais e sintomas compatíveis com reação alérgica, tais como prurido e lesões de pele.

Entre as variáveis estudadas, o sexo, o sistema $A B O$, o fator Rh e os valores de $\mathrm{Hb}$ e $\mathrm{Ht}$ não se mostraram associados à gravidade das reações transfusionais imediatas. Somente a idade $(p=0,003)$ e a unidade de ocorrência $(p=0,001)$ foram estatisticamente significantes pelo teste $x^{2}$ de Pearson. Os pacientes com reações mais graves eram mais velhos, e a proporção de reações de gravidade moderada/grave foi maior $(24,0 \%)$ nas unidades críticas e semicríticas. Em outros dois estudos, o primeiro conduzido no Irã, não houve associação entre a idade e a ocorrência de reação transfusional ${ }^{18}$, e o segundo conduzido em Cuba, não houve nenhuma reação leve em unidades de terapia intensiva/ críticas, com $8,0 \%$ das reações consideradas graves ${ }^{25}$.

\section{CONCLUSÕES}

A análise dos dados coletados rotineiramente na instituição possibilitou avaliar a prática transfusional, que se mostrou bastante adequada, refletindo os constantes esforços de identificação e notificação dos incidentes transfusionais. Os resultados evidenciaram como fatores de risco pacientes com 50 ou mais anos internados nas unidades críticas e semicríticas com doenças crônicas ou câncer, submetidos à terapia transfusional, de forma a prevenir complicações relacionadas a estes procedimentos. Em relação à gravidade, observa-se maior frequência de reações graves e moderadas nas unidades críticas, contudo as equipes de saúde não podem negligenciar cuidados com as reações leves que ocorrem em outras unidades.

Ao avaliar grande quantidade de hemotransfusões ao ano, este estudo pode contribuir para subsidiar ações de hemovigilância no Brasil, que ainda são incipientes.

Apesar dos esforços em monitorar, investigar e notificar as reações adversas relacionadas às transfusões sanguíneas, ainda pode haver subnotificação desses eventos na instituição pesquisada, assim como em outros serviços de hemoterapia brasileiros. Não foi possível investigar a morbimortalidade das reações transfusionais com seguimento dos pacientes expostos à hemotransfusão. Dessa forma, sugerem-se novas abordagens para avaliar os fatores de risco associados às transfusões sanguíneas.

\section{REFERÊNCIAS}

1. Macedo ED, Silveira VMJ, Athayde LA. Índice de reação transfusional em pacientes submetidos a transfusão em um hemocentro do norte de Minas Gerais. Rev Bras Pesqui Cienc Saude. 2015;2(2):54-9.
2. Agência Nacional de Vigilância Sanitária - Anvisa. Marco conceitual e operacional de hemovigilância: guia para hemovigilância no Brasil. Brasília: Agência Nacional de Vigilância Sanitária; 2015. 
3. Sahu S, Hemlata, Verma A. Adverse events related to blood transfusion. Indian J Anaesth. 2014;58(5):543-51. https://doi.org/10.4103/0019-5049.144650

4. Grandi JL, Grell MC, Barros MO, Chiba AK, Barbosa DA. Frequência dos incidentes transfusionais imediatos em receptores de hemocomponentes. Vigil Sanit Debate. 2017;5(2):83-8. https://doi.org/10.22239/2317-269X.000878

5. Beserra MPP, Portela MP, Monteiro MP, Façanha MC, Adriano LS, Fonteles MMF. Transfusion reactions in a hospital accredited in Ceará: an approach in hemovigilance. Arq Med. 2014;28(4):99-103.

6. Reis VN, Paixão IB, Perrone ACASJ, Monteiro MI, Santos KB. Transfusion monitoring: care practice analysis in a public teaching hospital. Einstein. 2016;14(1):41-6. https: / / doi.org/10.1590\%2FS1679-45082016AO3555

7. Guglin N, Zucker MJ, Bazan UM, Bozkurt B, Banayosy AE, Estep JO et al. Venoarterial ECMO for adults. J Am Coll Cardiol. 2019;73(6):698-716. https://doi.org/10.1016/j.jacc.2018.11.038

8. Paula IC, Azevedo LCP, Falcão LFR, Mazza BF, Barros MMO, Freitas FGR et al. Perfil transfusional em diferentes tipos de unidades de terapia intensiva. Rev Bras Anestesiol. 2014;64(3):183-9. https://doi.org/10.1016/j.bjan.2013.07.012

9. Handel J, Lang EA. Transfusion strategies for acute upper gastrointestinal bleeding. CJEM. 2015;17(5):582-5. https://doi.org/10.1017/cem.2014.76

10. Santos JP, Alzamora A, Teles MD, Lucena V, Paula TAO, Del Padre TCG et al. Transfusion reaction and hemovigilance: an imperative discussion in brazilian hemotherapy services. Int Arch Med. 2017;10:1-10. https://doi.org/10.3823/2526

11. Ballester $S$, Hernandéz AAB, Fernandéz RL, Rodriguez $M B$, Santovenia JMB. La hemovigilancia de las reacciones adversas a la donación de sangue. Rev Cuba Hematol Inmunol Hemoter. 2013;29(2):154-62.

12. Centro de Vigilância Sanitária - CVS. Boletim de hemovigilância 2. São Paulo: Centro de Vigilância Sanitária; 2016.

13. Berro $M$, Insagaray J, Barindelli $P$, Sosa $E$, Marcalain V, González J et al. Implementación de un sistema de hemovigilancia en el hospital de clínicas de Uruguay. Rev Med Urug. 2016;32(4):268-73.

14. Macedo ED, Silveira VMJ, Athayde LA. Índice de reação transfusional em pacientes submetidos a transfusão em um hemocentro do norte de Minas Gerais. Rev Bras Pesqui Cienc Saude. 2015;2(2):54-9.
15. Saito M. Hemovigilância: eventos transfusionais adversos antes e após a implantação do comitê transfusional hospitalar [dissertação]. Londrina: Universidade Estadual de Londrina; 2010[acesso 20 ago 2017]. Disponível em: http://www.uel.br/pos/saudecoletiva/Mestrado/diss/114.pdf

16. Chung KW, Harvey A, Basavaraju SV, Kuehnert MJ. How do hospitals participate in national recipient hemovigilance in the United States? Transfusion. 2015;55(4):703-7. https://doi.org/10.1111/trf.12980

17. Melians Abreu SM, Esquivel Hernández M, Padrino González M, Martín Álvarez I. Hemovigilancia y uso óptimo de los componentes en el ámbito hospitalario. Rev Ciencias Medicas. 2016;20(4):108-22.

18. Azizi S, Tabary SZ, Soleimani A. Prevalence of acute blood transfusion reactions in mazandaran heart center, Sari, Iran, 2010-2012. Med Arch. 2014;68(2):137-9. https://doi.org/10.5455/medarh.2014.68.137-139

19. Kato H, Nakayama T, Uruma M, Okuyama Y, Handa $M$, Tomyama $Y$ et al. A retrospective observational study to assess adverse transfusion reactions of patients with and without prior transfusion history. Vox Sanguinis. 2015;108(3):243-50. https://doi.org/10.1111/vox.12208

20. Souza GF, Nascimento ERP, Lazzari DD, Böes AA, lung W, Bertoncello KC. Good nursing practices in the intensive unit care: care practices during and after blood transfusions. Rev Min Enferm. 2014;18(4):939-46. http://www.doi.org/10.5935/1415-2762.20140069

21. Dupuis C, Sonneville R, Adrie C, Gros A, Darmon M, Bouadma $L$ et al. Impact of transfusion on patients with sepsis admitted in intensive care unit: a systematic review and meta-analysis. Ann Intensive Care. 2017;7:1-13. https://doi.org/10.1186/s13613-016-0226-5

22. Agência Nacional de Vigilância Sanitária - Anvisa. Boletim de produção hemoterápica: Hemoprod 2014 e 2015. 4a ed. Brasília: Agência Nacional de Vigilância Sanitária; 2015.

23. Mast AE, Steele WR, Johnson B, Wright DJ, Cable RG, Carey $P$ et al. Population-based screening for anemia using first-time blood donors. Am J Hematol. 2012;87(5):496-502. https://doi.org/10.1002/ajh.23171

24. Grandi JL, Grell MC, Areco KCN, Barbosa DA. Hemovigilância: a experiência da notificação de reações transfusionais em hospital universitário. Rev Esc Enferm USP. 2018;52:1-7. https://doi.org/10.1590/S1980-220X2017010603331

25. Martinez AM, Jimenéz RAR, Delgado NF. Efectos adversos en la cadena transfusional en el Instituto de Hematología e Inmunología. Rev Cubana Hematol Immunol Hemoter. 2015;31(3):288-300.

\section{Contribuição dos Autores}

Grandi JL - Concepção, planejamento (desenho do estudo), análise, interpretação dos dados e redação do trabalho. Chiba A, Oliveira MMB, Areco KCN - Aquisição, análise e interpretação dos dados. Barbosa DA - Redação do trabalho. Todos os autores aprovaram a versão final do trabalho. 